\title{
ED Acuity in Patients with Respiratory Complaint During the COVID-19 Pandemic: A Single-center Experience
}

\author{
Michael Anthony Abala ${ }^{1}$, Simranjeet Benipal ${ }^{1}$, Andrew Shen ${ }^{2}$, Jose Puglisi ${ }^{1}$, and Leonard Ranas- \\ inghe ${ }^{1 *}$
}

${ }^{1}$ College of Medicine, California Northstate University, USA

${ }^{2}$ Department of Emergency Medicine, Alhambra Hospital Medical Center, USA

*Corresponding author: Leonard Ranasinghe, Professor of Medical Education \& Emergency Medicine, California North state University College of Medicine, 9700 West Taron Drive, Elk Grove, USA

To Cite This Article: Leonard Ranasinghe, ED Acuity in Patients with Respiratory Complaint During the COVID-19 Pandemic: A Single-center Experience. 2020 - 9(6). AJBSR.MS.ID.001454. DOI: 10.34297/AJBSR.2020.09.001454.

Received: 酸July 19, 2020; Published: 制 August 14, 2020

\begin{abstract}
Introduction: In response to the novel coronavirus (COVID-19) pandemic, "shelter-in-place" (SIP) orders were implemented across the country to control the spread of this respiratory infection and decrease the burden on hospitals in the United States. Our objective was to assess changes in emergency department (ED) volume and acuity in patients with a respiratory complaint in a single center in Los Angeles county, California during the pandemic with respect to the SIP order.

Methods: We conducted a single-center retrospective review that included de-identified data from patients presenting to the ED with a respiratory complaint from December 2019 to May 2020. We examined the monthly distribution of triage levels, as defined by the Emergency Severity Index, as an approximation of general acuity and resource utilization. We also examined the monthly ED volumes of patients presenting with a respiratory complaint and its relationship to overall ED volume. We then compared these data in the periods before and after the implementation of SIP in Los Angeles county, California on March 23, 2020.
\end{abstract}

Results: A significant decrease in absolute (56\%) and relative ED volume (12.7\% to 10.4\%) in patients with respiratory complaint post-SIP declaration was observed $(\mathrm{p}<0.01)$. The distribution of patient triage levels exhibited a significant shift towards increased acuity and resource utilization $(\mathrm{p}<0.01)$.

Conclusion: Fewer patients with a respiratory complaint came to the ED after its implementation at this site. Despite this, increased acuity was observed after the SIP order was enacted, suggesting that those coming to the ED for respiratory complaints were generally sicker and needed more resources to be properly cared for.

\section{Introduction}

Amidst the novel coronavirus (COVID-19) pandemic, respiratory disease has garnered newfound attention from the public. Historically, respiratory diseases, such as COVID-19, have placed a significant burden on the United States (US) healthcare system by affecting millions of people nationwide and costing the country billions of dollars in indirect costs and direct health care expenditures [1,2]. As of June 18, 2020, over 2.1 million cases have been reported in the US with a mortality rate of 5.46\% [3]. The widespread impact of COVID-19 is attributed to its rapid spread through contact, airborne, and droplet transmission [4,5].

Data from the World Health Organization (WHO) reports that the first human cases of COVID- 19 occurred in early December 
2019 in Wuhan, China. On January 20th, 2020, the first case of COVID-19 infection in the United States was reported in Snohomish County, Washington [6]. On March 13th, 2020, a state of national emergency was declared in the US in response to the global COVID-19 pandemic [7]. Soon, several states began enforcing statewide "Shelter-In-Place" (SIP) orders to attempt to mitigate the spread of COVID-19 infection. In the weeks that followed, hospitals across the nation began to experience sharp drops $(\sim 42 \%)$ in the volume of patients presenting to the emergency department (ED) [7].

However, speculation has arisen on the effects of the COVID-19 pandemic on the acuity of patient cases, which corresponds to the condition severity and the ED resource utilization per patient [8]. Limiting the spread of this respiratory virus should result in reduced need for emergency respiratory services. On March 23, 2020, SIP orders were imposed in Los Angeles county, which our hospital-of-focus serves and where most of the COVID-19 cases have been reported in California [9]. As COVID-19 patients present with primarily respiratory symptoms [10], our objective was to assess changes in ED volume and acuity in patients with respiratory complaint before and after the implementation of the SIP order.

\section{Methods}

\section{Data Collection}

This was a retrospective study conducted using appointment data $(n=20,390)$ from the Alhambra Hospital Medical Center, a single, large community hospital in Los Angeles County, California, that handles over 15,000 ED visits a year. Patient data was deidentified and extracted from the institution's electronic health records with the intent of quality improvement; institutional review board approval was not required. We identified ED visits $(n=2,172)$ presenting primarily with a respiratory chief complaint from December 1st, 2019 to May 31st, 2020. This timeframe roughly approximates when the first coronavirus case was reported in China to the end of the SIP order in Los Angeles County.

Monthly triage levels, patient volumes, dispositions, and timestamps were collected, as seen in Table 1. Triage levels were reported ordinally from " 5 " to " 1 " per the Emergency Severity Index (ESI) [8]. These levels approximated the general acuity of each case, with increased acuity and increased utilization of ED resources as values neared " 1 ".
Each category is elaborated as follows:

a. Level 5: No ED resources were needed, low risk/distress

b. Level 4: One ED resource was needed, low risk/distress

c. Level 3: Many ED resources were needed, low risk/ distress

d. Level 2: High risk, confused/lethargic, severe pain/ distress

e. Level 1: Required immediate life-saving intervention

Furthermore, in Table 1, ED volume represents patients presenting with a respiratory complaint. The relative percentage of cases with respiratory complaint in respect to the rest of the ED is also reported. ED disposition was subdivided into admitted, discharged, or other. Lastly, timestamps included arrival to bed, registered nurse, doctor, and discharge (length of stay) reported in minutes.

\section{Data Analysis}

We compared monthly triage levels, patient volumes, dispositions, and timestamps relative to all patients with a respiratory complaint during this timeframe. Data collected was further analyzed before and after March 23, 2020, the day that SIP was declared in Los Angeles County. We used the SPSS statistics software v.25 to analyze the raw data and determined statistical significance using chi-squared test, t-test, and Mann-Whitney U test. A p-value less than 0.05 was considered statistically significant.

\section{Results}

As seen in Table 1, a significant decrease (54\%) in overall ED volume post-SIP declaration on March 23, 2020 was observed $(\mathrm{p}<0.01)$. Respiratory patient volumes also significantly decreased from an average of $12.7 \%$ of all ED cases to $10.4 \%(p<0.01)$. Additionally, the proportion of patient admissions per month significantly increased $(\mathrm{p}<0.001)$, while the proportion of patients discharged significantly decreased $(\mathrm{p}<0.001)$ post-SIP. ED timestamps did not show a significant change pre- and post-SIP.

Figure 1 illustrates the distribution of triage levels for respiratory patients in the ED pre- and post-SIP. The distribution displayed a significant right-ward shift $(\mathrm{p}<0.01)$, indicating a change towards increased case severity.

\begin{tabular}{|c|c|c|c|c|c|c|}
\hline Table 1: ED Volume, Triage Level, Disposition, and Important Timestamps during COVID-19 Pandemic (Respiratory Complaint) \\
\hline & 19-Dec & 20-Jan & 20-Feb & 20-Mar & 20 -Apr & $20-M a y$ \\
\hline ED Volume (\%) & $210(11.6 \%)$ & $251(12.1 \%)$ & $226(12.8 \%)$ & $207(15.0 \%)$ & $102(12.5 \%) \dagger$ & $90(8.4 \%) \dagger$ \\
\hline Triage Level $^{\mathrm{b}}$ & & & & & & \\
\hline 5 & 9 & 17 & 12 & 16 & 2 & 1 \\
\hline
\end{tabular}




\begin{tabular}{|c|c|c|c|c|c|c|}
\hline 4 & 49 & 77 & 59 & 66 & 16 & 9 \\
\hline 3 & 108 & 117 & 124 & 103 & 58 & 53 \\
\hline 2 & 28 & 28 & 24 & 18 & 21 & 13 \\
\hline 1 & 3 & 1 & 0 & 1 & 2 & 3 \\
\hline Unassigned & 13 & 11 & 7 & 3 & 3 & 11 \\
\hline \multicolumn{7}{|l|}{ Disposition } \\
\hline Admitted (\%) & $49(23.3 \%)$ & $53(21.1 \%)$ & $62(27.4 \%)$ & $52(25.1 \%)$ & $42(41.2 \%) \ddagger$ & $31(34.4 \%) \ddagger$ \\
\hline Discharged (\%) & $147(70.0 \%)$ & $188(74.9 \%)$ & $155(68.6 \%)$ & $149(72.0 \%)$ & $54(52.9 \%) \neq$ & 53 (58.9\%)‡ \\
\hline Other $(\%)^{\mathrm{c}}$ & $14(6.7 \%)$ & $10(4.0 \%)$ & $9(4.0 \%)$ & $6(2.9 \%)$ & $6(5.9 \%)$ & $6(6.7 \%)$ \\
\hline \multicolumn{7}{|l|}{ Timestamps $^{\mathrm{d}}$} \\
\hline Arrival to Bed (IQR) & $12(8-21)$ & $13(8-27)$ & $12(7-21)$ & $11(6-19)$ & $9(3-14)$ & $10.5(7-16)$ \\
\hline Arrival to RN (IQR) & $14(9-22.25)$ & $15(9-29)$ & $13(8-22)$ & $12(7-20)$ & $10(4.5-16.5)$ & $11(7-16.75)$ \\
\hline Arrival to Doctor (IQR) & $8(12-20.25)$ & $14(8-26)$ & $12(6-23)$ & $11(6-22)$ & $9(4-15)$ & $10(6-15.75)$ \\
\hline Length of Stay (IQR) & $135(91-203.5)$ & 141 (91-209) & 132 (77-197) & $128(80-194)$ & $165(117-269.5)$ & $142.5(94.5-232.75)$ \\
\hline
\end{tabular}

Abbreviations: ED (emergency department); IQR (interquartile range between 1st and 3rd quartile); RN (registered nurse)

a-Percentage represents number of patients with respiratory complaint relative to total number of patients in ED for each respective month

b-Based on Emergency Severity Index, organized from no resources used (5) to life-saving treatment (1)

c-Neither discharged nor admitted (against medical advice, cancelled, eloped, expired, left after triage, left without being seen, or transferred)

d-Observed median reported in minutes

†0.01

$\neq \mathrm{p}<0.001$

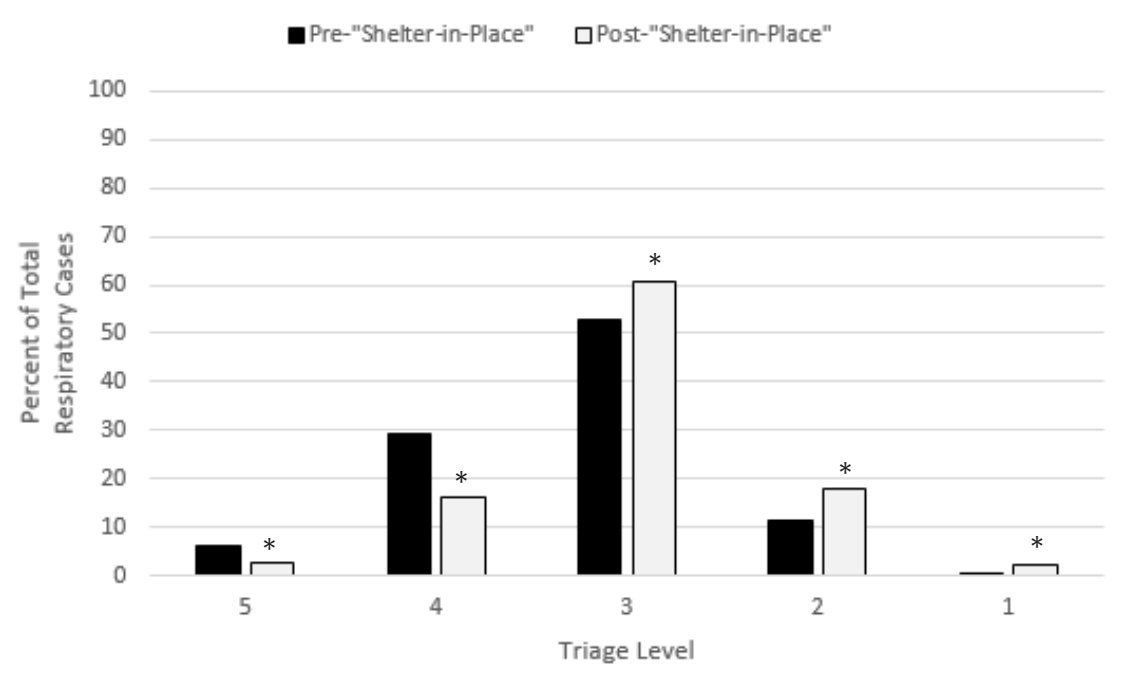

Figure 1: Triage level breakdown of respiratory cases before and after the implementation of "Shelter-in-Place" on March $23,2020$.

\section{Discussion}

The implementation of the SIP order on March 23, 2020 in Los Angeles county, California in response to the COVID-19 pandemic correlated with a significant decrease in both the volume of patients with respiratory complaint (56\%) and overall, ED volume (54\%). This finding is consistent with the current data done by the Centers for Disease Control (CDC) stating that ED's across the US have seen a $42 \%$ average decrease in patient volume [7]. The distribution of the triage level of respiratory cases shifted significantly towards increased acuity post-SIP. So, although the absolute number of cases diminished, an ED patient presenting with a respiratory complaint was expected to be in a more severe condition than those coming in pre-SIP on average in our study. 
In our study, we also saw a significant decrease in monthly admissions $(32.4 \%)$ with a larger significant decrease in nonadmissions (66.5\%), which may be accounted for by the decrease in ED volume. This supports that the SIP order helped decrease the burden placed on this ED in our study. Our investigation emphasizes that ED's should be properly supplied during this pandemic and prepared for the increased likelihood that a patient coming in will need to be admitted $(+13.9 \%)$ and may be suffering from a more serious condition $(p<0.01)$ than previously expected.

Recent data shows a 44\% increase in the COVID-19 hospitalization rate in California, from June 24, 2020 to July 8, 2020 [11]. With the sharp rises in COVID-19 cases across a reopening US, there is a newfound worry on the capability of US ED's in handling this pandemic since SIP orders were lifted. Governor Newsom claims that the current spike is attributed to the lifting of the SIP orders and decreased social distancing [11]. Our investigation and current modelling studies support the effectiveness of the SIP order in decreasing the burden placed on ED's across the country [12].

Though these stark differences were noted after the SIP order was enacted, we postulate that this decrease may also be correlated with the time of increased public awareness of the pandemic. This, in turn, may have caused decreased public utilization of ED's due to fear of contracting the COVID-19 infection with only those in more severe condition expected to come into the ED. Timestamps approximate the course of patient visits. Although the changes were not significant before and after SIP, the timestamp data presented in Table 1 can be used to set a patient's expectations for when they will receive a bed, first see a registered nurse and doctor, and how long they will be in the ED. This may mitigate fear in a patient with a respiratory complaint on if they should or should not go to the ED.

\section{Limitations}

Our results should be interpreted in the context of some limitations. First, this is a single-center study and may not be generalizable to other patient populations and locations that may have been impacted differently from COVID-19. Second, although COVID-19 patients primarily present with a respiratory complaint, a small subset of these patients do not present with a respiratory complaint [10]. However, our investigation remains useful in drawing insight into anticipated resource usage in a large subset of patients amidst a pandemic as well as the impact of a SIP order.

\section{Conclusion}

The SIP order enacted in Los Angeles county, California on March 23, 2020 showed that fewer patients with a respiratory complaint came to the ED after its implementation at this site. Despite this, increased acuity was observed after the SIP order was enacted, suggesting that those coming to the ED for respiratory complaints were generally sicker and more needed more resources to be properly cared for. This study illustrated the efficacy of a SIP order and its effect on resource utilization. Further research is warranted in examining ED volumes and acuity after SIP orders continue to be lifted.

\section{References}

1. Guarascio AJ, Ray SM, Finch CK, Self TH (2013) The clinical and economic burden of chronic obstructive pulmonary disease in the USA. Clinicoecon Outcomes Res 5: 235-245.

2. (2020) Respiratory Diseases. Respiratory Diseases | Healthy People.

3. Coronavirus Disease 2019 (COVID-19) in the U.S. Centers for Disease Control and Prevention.

4. Infection prevention and control during health care when novel coronavirus $(\mathrm{nCoV})$ infection is suspected. World Health Organization.

5. Bahl P, Doolan C, de Silva C, Chughtai AA, Bourouiba L, et al. (2020) Airborne or droplet precautions for health workers treating COVID-19? J Infect Dis jiaa189.

6. Holshue ML, DeBolt C, Lindquist S, Kathy H Lofy, John Wiesman, et al. (2020) First Case of 2019 Novel Coronavirus in the United States. N Engl J Med 382(10): 929-936.

7. Hartnett KP, Kite-Powell A, DeVies J, Tegan K Boehmer, Jennifer, et al. (2020) Impact of the COVID-19 Pandemic on Emergency Department Visits-United States, January 1, 2019-May 30, 2020. MMWR Morb Mortal Wkly Rep 69(23): 699-704.

8. Gilboy N, Tanabe P, Travers D, Rosenau A (2011) Emergency Severity Index (ESI): A Triage Tool for Emergency Department Care, Version 4. Implementation Handbook 2012 Edition. Rockville, MD: AHRQ.

9. Coronavirus Disease 2019 (COVID-19): Cases \& Deaths by County. Centers for Disease Control and Prevention.

10. Yuki K, Fujiogi M, Koutsogiannaki S (2020) COVID-19 pathophysiology: A review. Clin Immunol 215: 108427.

11. California hospitals prepare for influx of COVID-19 patients. KCRA Staff.

12. Nussbaumer-Streit B, Mayr V, Dobrescu AI, Andrea Chapman, Emma Persad, et al. (2020) Quarantine alone or in combination with other public health measures to control COVID-19: a rapid review. Cochrane Database Syst Rev 4(4): CD013574. 